\title{
Measuring Single Electrons - What Does it Mean?
}

Henning Ryll ${ }^{*}$, Martin Huth ${ }^{2}$, Robert Ritz ${ }^{2}$, Colum M. O'Leary ${ }^{3}$, Ian Griffiths ${ }^{3}$, Peter Nellist ${ }^{3}$, Ryusuke Sagawa $^{4}$, Yukihito Kondo ${ }^{4}$, Heike Soltau ${ }^{2}$ and Lothar Strüder ${ }^{1}$

1. PNSensor GmbH, Otto-Hahn-Ring 6, 81739 München, Germany

2. PNDetector GmbH, Otto-Hahn-Ring 6, 81739 München, Germany

3. Department of Materials, University of Oxford, 13 Parks Road, Oxford OX13PH, UK

4. JEOL Ltd., 3-1-2 Musashino Akishima Tokyo 196-8558, Japan

* Corresponding authors: henning.ryll@pnsensor.de

In today's electron microscopy research, a strong trend towards ultra-low dose experiments is driven by challenging samples, which are beam-sensitive and may charge or deteriorate during the measurement process. Recent experiments indicate that only few electrons passing through the sample already carry enough information to deduce sensitive information like electric or magnetic field distributions. Even quantitative phase contrast imaging is possible with sub-pA beam currents.

In such context, the capability of measuring single electrons is the ultimate goal and various detectors claim to achieve this. However, the readout schemes of pixelated direct detectors available differ dramatically in how the energy of the primary electron, which is deposited in the bulk silicon, is converted to intensity signal which is finally stored in the data. Here, the complex electron - silicon interactions need to be accounted for in order to create valid data.

In this work we present unprocessed true single electron data, together with electron track simulations in silicon (Fig. 1a, 1b). Combining the real analogue detector response to electron impingement and sophisticated event analysis achieves a high accuracy in finding the original entry point, while the true number of electrons per diffraction pattern is intrinsically contained in the analogue data. Here, even the effect that some of the primary electrons hitting the detector only leave a fraction of their full energy in the detector (backscattered electrons) can be accounted for.

The data shown is recorded with our ultrafast pixelated direct detector, the pnCCD (S)TEM camera [1] (Fig 2). In addition to the excellent energy sensitivity of the detector, a high spatial resolution, here 264 x 264 pixels, is crucial in order to know "how many" electrons hit the detector "where", and finally form the diffraction pattern.

A discussion is presented by evaluating the effects of preprocessing the detector response by applying thresholds to true analogue data (Fig. 1c) and simulated counting detector data.

\section{References:}

[1] Ryll, H., et al, Journal of Instrumentation 11 (2016) 

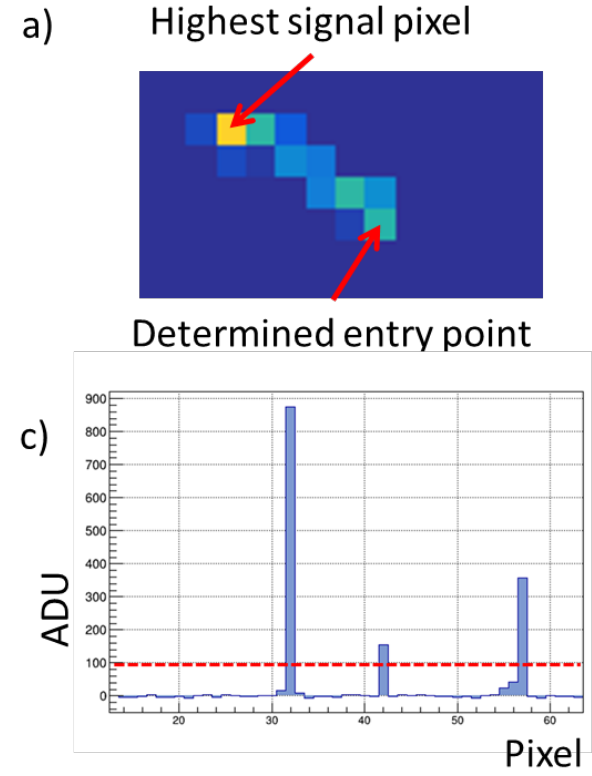

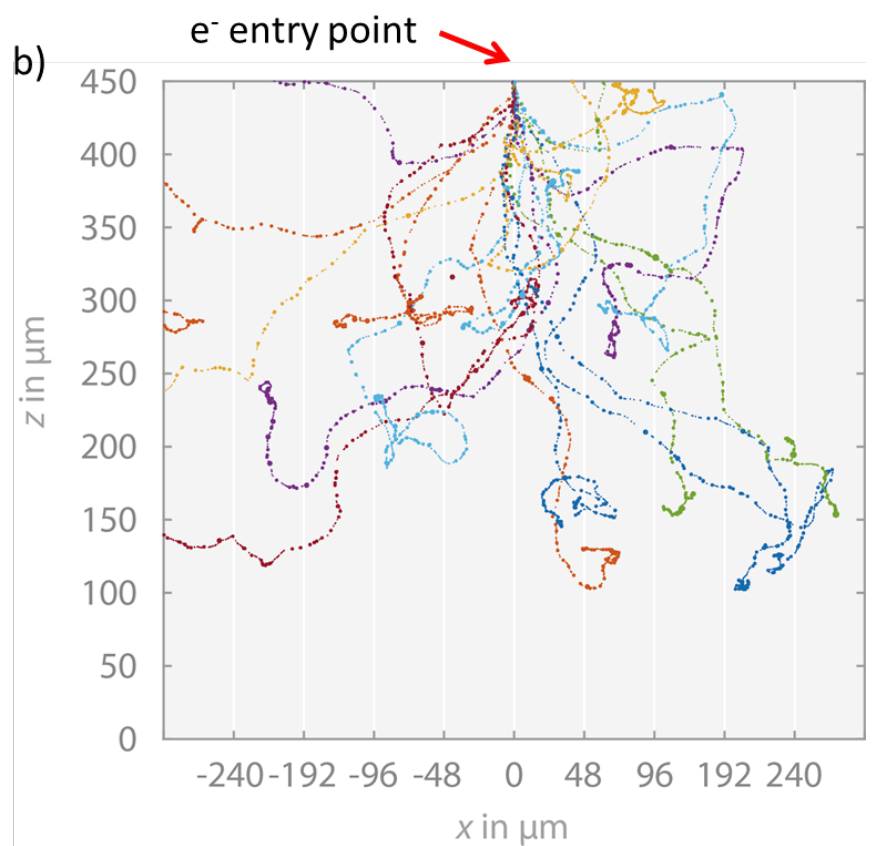

Figure 1. a) Measured track of a single $300 \mathrm{keV}$ electron. b) Side cut view of several simulated tracks of $300 \mathrm{keV}$ electrons all entering at the same point. c) Intensity line profile along one detector channel crossing the signal from three primary electrons. Red dotted line represents a possible threshold position and is shown for visualizing discussed topics about thresholding.
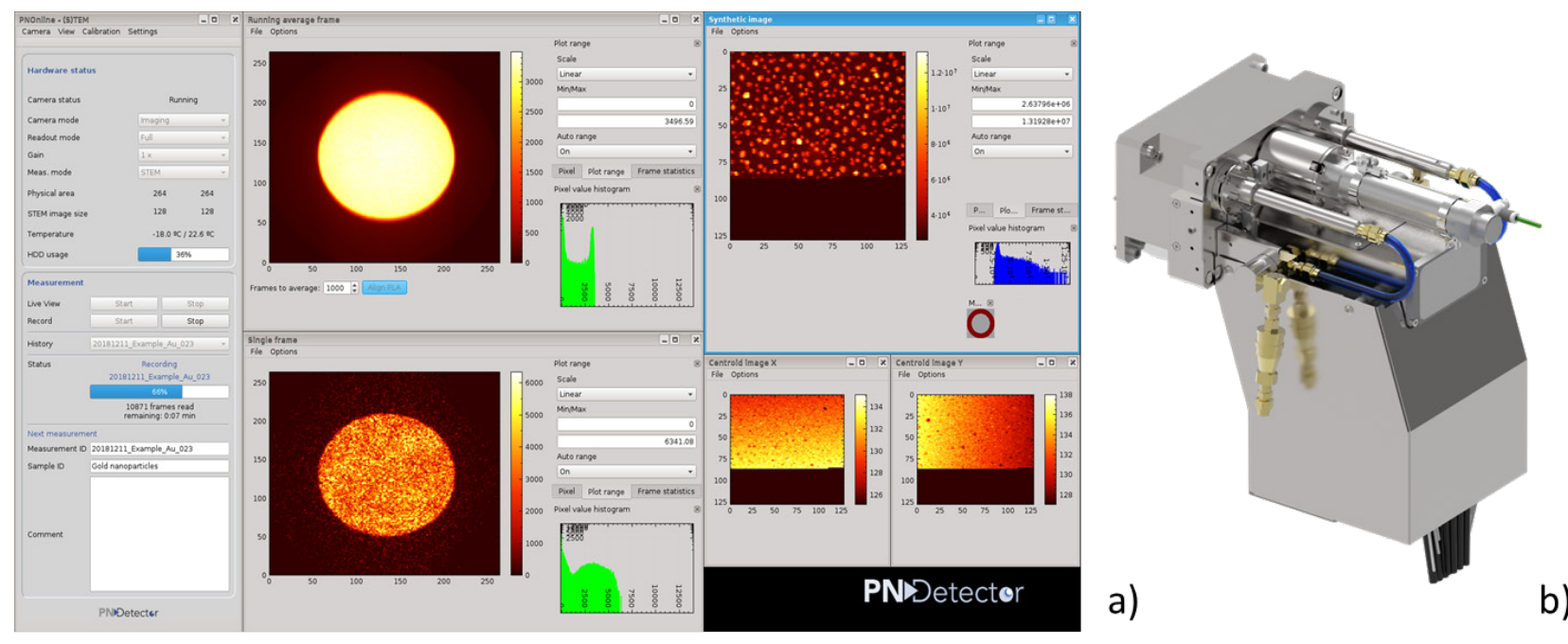

Figure 2. a) Software "PNOnline" for data acquisition and controlling the 4D Canvas camera. b) Picture of the 4D Canvas Detector, which is the JEOL integrated version of the pnCCD (S)TEM camera. 